\title{
Family-line and Socioeconomic Factors in Fatness and Obesity
}

\author{
Stanley M. Garn, PhD
}

$\mathbf{T}$ here are hundreds of explanations for why one individual is fatter than another, and why some people are truly obese. Explanations range from the sensory (involving differences in taste and olfactory acuity), to neuroendocrine, to metabolic, to the mode and amount of feeding in early infancy, to the composition of the diet. To some, obesity represents an ancient adaptation to cyclical fluctuations in food availability. To others, obesity simply reflects a lack of willpower or the summed results of food availability and decreased opportunities for energy expenditure. There is some evidence to support each of these explanations and dozens of others, including failures in the appetite-regulating mechanisms and individual differences in the deposition patterns of nonpigmented fat.

Of all the data amassed on fatness levels and on obesity, as variously defined, two sets of observations command the most attention. The first is that above-average fatness and obesity are concentrated in particular families, ie, that fatness runs along family lines. ${ }^{1}$ The child of one obese parent is more likely to be obese, ${ }^{2}$ increasingly so if both parents are obese, ${ }^{3}$ even more so if the grandparents are obese as well. ${ }^{4}$ The second observation has to do with socioeconomic status (SES) as measured by income, education, and occupation. Though poorer children of both sexes are leaner than their more affluent peers, ${ }^{5,6}$ the

Dr Garn is a Fellow, Center for Human Growth and Development; Professor of Nutrition, School of Public Health; and Professor of Anthropology, The University of Michigan, Ann Arbor, MI 48109. relationship between socioeconomic status and fatness is inverse among adult women, often dramatically so. ${ }^{7}$ At age 50 the impoverished woman averages $8 \mathrm{~kg}$ more in weight than the higher-income woman, yet the poorer woman is shorter. 8,9

The existence of positive parent-child correlations in fatness is unquestionable, given tens of thousands of observations. The magnitudes of the mother-child or father-child correlations approximate 0.25 , as might be expected for polygenic inheritance. ${ }^{10}$ Sibling correlations for measured fatness (subscapular, triceps, iliac, or abdominal) are also positive and somewhat higher than 0.25 , as would be expected assuming the genetic hypothesis. ${ }^{10}$ If we make use of parental fatness pairings from lean $x$ lean through obese $x$ obese, and including the intermediate categories (medium $x$ medium, medium $x$ lean, medium $x$ obese), the fatness levels of their children increase in agreeably stepwise fashion, achieving textbook perfection. ${ }^{11}$ One might assume that the level of fatness is hereditary and additive, were it not for still other observations.

One additional observation is that spouses also resemble each other in fatness levels, which might be due to selective mating, but which could also be due to years of living and eating together. A second observation is that parent-child and sibling correlations in fatness actually increase during the growing years, which could represent timing effects, but might also reflect the cumulative effects of communal living. A third observation is that parent-child fatness correlations decrease after the children become adults and are no longer living with their parents. ${ }^{12} \mathrm{~A}$ fourth observation is that 
family members still living together show synchronic fatness changes, ie, they go up and down in fatness level together. ${ }^{13}$ Since mothers and fathers are genetically unrelated individuals and average more than 2 decades older than their children, it is difficult to ascribe synchronic fatness changes to genes held in common. As with decreased fatness correlations when the generations no longer live together, nongenetic and environmental effects must be given serious attention.

The alternative model to genetically related individuals living together is either 1) genetically unrelated individuals living together, or 2) genetically related individuals living apart. Spouses are one example of the first alternative model, and there are systematic spousal similarities in fatness. Adopted children compared with adoptive parents also comprise examples of the first alternative model and they too show similarities in fatness. ${ }^{14,15}$ This is contrary to genetic expectations, but there are complications introduced by the age at adoption, the duration of adoption, the type of adoption, and even seasonal fluctuations in fatness levels which may inflate apparent fatness similarities.

The second alternative model, that of genetically related individuals living apart, is well represented by the data from the Danish Adoption Study, involving older parents and their genetic progeny studied as adults. ${ }^{16}$ Though the selfreported weight-for-height data do suggest similarities in these long-separated parentchild pairs, there are limitations in weight-forheight or the Body Mass Index (wt/ht ${ }^{2}$. Weight-for-height is affected by relative leg length (or body proportions) and it measures both the lean body mass and the weight of fat to equal degree. Therefore, the older Danish parents and their long-separated adult children may be similar in fatness, or the weight-forheight data may reflect similarities in body proportions and the size of the lean body mass. Many workers have challenged weight-forheight as a valid measure of adiposity. ${ }^{17,18}$

In theory, fatness comparisons of monozygotic and dizygotic twins should do much to resolve the nature-nurture controversy and should provide numeric estimates of the heritability of human fatness, symbolized by $\mathrm{H}^{2}$. In practice, however, such comparisons have been disappointing, for technical (methodological) and other reasons. Since the reliability of fatness measurements is only \pm 5 percent at best, actual monozygotic twin correlations necessarily fall below the theoretic maximum of 1.00. Dizygotic twin correlations for fatness should approximate those for singleton siblings but, in practice, are somewhat higherup to 0.50 -indicating that environmental factors tend to inflate all twin (and triplet) fatness resemblances. ${ }^{19}$ Moreover, the age effect again comes into play, with monozygotic twins becoming less alike in fatness as they reach adulthood, not infrequently differing by \pm 1 Z-score (ie, standard deviation unit) or more. The most recent studies and data analyses made in Canada, estimating the genetic and environmental sources of fatness variability, tend to give common genes second place to environment when it comes to fatness, besides indicating the need for very large samples as well. ${ }^{20}$ American twin-registering studies again show that monozygotic twins' similarities are less than perfect. ${ }^{21}$ Possibly, the ultimate editorial comment on fatness determinants comes from Mason's often-cited observation that obese adults and elderly adults in England tend to be owners of obese pets. ${ }^{22}$

One assumption often made in fatness studies is that higher levels of fatness are more or less constant, meaning that the obese remain obese over prolonged periods of time. This assumption is compromised to some extent by the normal volatility of fatness on the part of the obese, which may be considerable over a 1year period. ${ }^{23}$ Some of the obese are gaining in fatness and some are losing fatness at any given time. Yet when it comes to the long-term predictability of fatness, from infancy through to adulthood, nearly all investigators have come to the same conclusions. Obese infants, as variously defined, do tend to remain obese as adolescents or as adults, more often than chance would allow, ${ }^{24-26}$ with risk ratios or relative risks of 1.6 to 1.8. Yet most obese babies and infants do not mature into obese adults. The majority of obese adult males do not remain obese for 2 decades, either, though obesity in young adulthood is still a risk factor for middle-aged obesity in the male. ${ }^{27}$ The fact 
that the obese do not necessarily remain obese has been explained by the suggestion that there may be different types of obesity (juvenile-onset, adolescent-onset, and adultonset) with different causes and courses. It becomes important, therefore, to ascertain why some infants start obese and remain obese while most do not, why some obese adult males lose fatness level, and why most obese adult females do not.

\section{Maturational Timing and Fatness}

Given the volatility of fatness, the proportion of the obese who become less than obese and the proportion of lean individuals who later become obese, it is intriguing to find one biological variable that relates to fatness level, lifelong. That variable is menarcheal timing, ie, the age at menarche, which not only predicts fatness in the woman herself, but also predicts fatness in her offspring of both sexes. Early maturing women weigh more and are fatter by the third decade, and increase in weight and fatness thereafter, becoming $4 \mathrm{~kg}$ heavier and 30 percent fatter by the fourth decade. ${ }^{28-30}$ Even in the sixth and seventh decades, earlymaturing women are fatter than their late-maturing peers. Studied prospectively, both the sons and the daughters of early-maturing women are fatter in infancy, childhood, adolescence, and into adulthood-through to middle age. Obese boys and girls and men and women tend to be the progeny of early-maturing mothers. Lean boys and girls and men and women tend to be the sons and daughters of late-maturing mothers. Exactly how maternal maturity timing determines or controls fatness level is not clear, but the differences are considerable and they cross generational lines. It may be that maturity timing is one expression of the genetic part of fatness variance, and that it is the early-maturer who starts fat and stays fat, ie, the individual who "tracks" in relative fatness, lifelong. ${ }^{30}$

\section{Socioeconomic Aspects of Fatness and Obesity}

In contrast to the biologic determinants of fatness level and obesity, which reveal level after level of complexity, socioeconomic determinants of fatness level are simpler to de- scribe; they are based on still larger samples, but with surprising outcomes. Least surprising is the fact that impoverished boys and girls and poverty-level men are leaner in most countries and most populations studied. Whether defined by income, occupation, education, or house type, low SES is associated with smaller size and less fat in both boys and girls, compared with their more affluent peers of higher SES. Because food is costly, and because the poor have lower purchasing power arid often larger families, with less access to medical care and less stringent supervision of activity levels, energy intake and energy expenditure together would seem to be an adequate explanation for such worldwide differences in measured fatness and in the risk of being obese.

When we turn to adult women, and to girls older than age 15, SES is inversely rather than positively (or directly) related to fatness level. This is uniformly true in the massive data of the Ten-state Nutrition Survey (TSNS), the data of the National Health and Nutrition Examination Surveys (NHANES), and the data of the Tecumseh Community Health Studies. 6,9,27 In all these studies, and in other studies in Western countries, low-income women are heavier, fatter, and more often obese. More affluent women (though taller) are lighter in weight and leaner, often to a considerable degree. A 12-kg $(30-\mathrm{lb})$ difference in weight and a larger difference in fatness is not unusual in comparing poverty-level and above-median income groups of women in the middle years. Indeed, there is a striking inverse relationship between SES and fatness level in women.

These data may be restated by saying that poorer, leaner preadolescent girls become poorer, fatter adult women, while richer, fatter girls mature into richer, leaner women. It is difficult to explain this socioeconomic reversal of fatness in the female by invoking the sensory hypothesis, set-point theory, adipocyte size and number, or most genetic or physiologic explanations. Clearly, the richer women respond to the message of leaness carried by the print, picture, and electronic media, but why do the magazines, newspapers, and television messages fail to reach the poorer women? Why does the reversal take place at adolescence, around age 15 ? Why do more 
affluent women fit the oft-repeated dictum of the late Wallis Warfield Simpson ("No woman can be too rich or too thin"), and why do poor women fail to do so? Why are poor women at much greater risk of obesity despite their lower purchasing power? Why do low SES women fail to become "weight watchers"?

The direction (but not the magnitude) of the socioeconomic difference in fatness is the same for children the world around, whether SES is measured by Warner's ranks, income relative to needs, the Bureau of Census Socioeconomic Index, or house types and holdings. Poorer children from larger families with lower purchasing power, less knowledge of nutrition, and experiencing larger cyclical fluctuations in the food supply, are leaner and have lower lipid levels, serum and urinary vitamins, and serum albumin levels than their more affluent peers. They are also smaller, and mature later.

There has been some confusion in summarizing these socioeconomic differences in fatness, in part because the directions are different for children of both sexes and adult women, but not for adult men. If fatness is described as poverty-related, that statement must be explicit as to age and sex. There has also been confusion in generalizing the socioeconomic directions of fatness because American and English socioeconomic rankings are arranged in different orders (the English "l" corresponding to the American "high"), leading to seeming differences in direction. Still further confusion arises from comparing mean or median levels of fatness, or the percent deemed obese or the percent deemed superobese by socioeconomic level. A smaller socioeconomic difference in skinfold thickness may be associated with a seemingly large difference in the incidence of obesity, and a still larger difference in the proportion of individuals deemed superobese. As with the quantification of socioeconomic status, by index numbers or by ranks, the expression of fatness levels and differences can cause confusion even among experienced investigators.

Among American adults, the relationship between SES and fatness is curvilinear in both sexes, being lowest below the poverty level, then rising, then falling again. Among men, fatness levels increase through medium in- come levels; then they decrease. Among women, fatness levels peak at poverty level (at which point wives often weigh as much as their husbands); then fatness decreases as income or income relative to needs goes up. For the highest income levels, sparsely represented in national surveys, the level of fatness and the incidence of obesity is low in both sexes; the "beautiful people" of the society pages are generally lean. How the SES continuum is cut makes considerable difference to the results and to the generalizations that can be made.

\section{Socioeconomic Differences and Fatness Norms}

While socioeconomic differences in the level of fatness and the incidence of obesity, and the socioeconomic "reversal" of fatness in the female, raise questions of etiology and mechanisms, their existence and magnitude serve as useful warnings in diagnosing obesity and suggesting recommended or ideal levels of fatness. Population data, such as the data from the NHANES, include a mixture of poor and affluent Americans who differ in fatness level. Who provides better reference data for normative use, the poor or the rich? Since insurance data on mortality reflect the experience of a rather select group of insured white individuals, are the weight-for-height values associated with minimum long-term mortality applicable to other population segments? Since the percentiles for fatness in the various NHANES cycles include a mixture of leaner smokers and fatter nonsmokers, should we exclude the former in defining obesity? Since the 1959 and 1979 insurance data also comprise a mixture of leaner smokers and fatter nonsmokers, should these data be censored to exclude the smokers with their poorer life expectancy? Questions such as these, and their answers, are of great practical importance in deciding who is "too fat" and what level of fatness is ideal for fitness or long-term survival.

Socioeconomic differences in fatness also bear on sibling similarities in fatness during the growing years, on like-sexed sibling similarities during the adult years, and on similarities between adopted children as well. They bear also on black-white differences in fatness, explaining (in the statistical sense) the greater fatness 
of black women and the lesser fatness of black children and black males. The greater fatness of American Indian women is also explicable in terms of their lower income levels, and so are the higher birthweights of Cree Indian babies.

Most students of fatness and obesity accept the role of both genetic and social factors in determining the fatness level of any individual, refusing to be categorized as strict hereditarians or complete environmentalists just to please the working press. The question to be asked is not which factor is involved in fatness level, but how much of each, under the conditions of a given study. Despite the large fatness differences between poor women and rich women, and fatness differences between Americans and their cousins abroad, there is considerable interpersonal variance that can be genetic in nature. Despite the highest estimates of heritability, in particular studies, there is a considerable fraction of fatness variance attributable to nongenetic factors. But various estimates of the heritability of fatness vary from under 35 percent in one recent study ${ }^{31}$ to over 80 percent in another recent study; ${ }^{21}$ and twinstudy estimates were viewed as "unrealistic" in a third study. ${ }^{20}$ Therefore, fractionating the genetic and nongenetic components of fatness may still be more statistical than actual, even with "environment" specifically subtracted from the family-line estimates. ${ }^{32}$ Skinfolds, weight, and relative weight do yield different estimates. ${ }^{33}$ Indeed, many of the established investigators in the fatness field are known for their contributions in both directions, derived from different study samples and different investigative designs. While there are innumerable suggestions in the published literature as to how the obese and the lean differ from each other-in their frequency of eating, amounts of food consumed, or response to caloric deprivation-these differences may reflect the consequences of being obese and lean, and not their causes. (They may also reflect editorial policies that favor positive findings, but allow the 5-percent level of significance when many different measurements are compared.)

Despite the hard-core obese who are alleged to be resistant to all forms of therapy, including surgical intervention, it is encouraging to discover in population contexts that the majority of obese men do not remain obese. It is equally encouraging to learn that only a minority of "obese" infants mature into obese adults. The dynamics of fatness change, including synchronic fatness change among family members living together, may be more revealing than the statistics of fatness level, especially among obese individuals who are notably fatness-volatile. Obese members of nonobese families may offer better insight into the development of obesity than obese members of obese families with half-a-dozen relatives reinforcing their habits. Spontaneously obese monkeys in primate colonies may help our thinking, as may prisoners restricted to prison food, and more sophisticated experimental designs to separate the effects of living together and genes held in common. $\square$

1. S Matsuki and R Yoda, Keio J Med 20: 135-141, 1971

2. SM Garn and DC Clark, Pediatrics 56: 306-319, 1975

3. SM Garn and DC Clark, Pediatrics 57: 443-456, 1976

4. SM Garn, SM Bailey, MA Solomon, and PJ Hopkins, Am J Clin Nutr 34: 148-153, 1981

5. FE Johnston, PVV Hamill, and S Lemeshow, US Dept of Health, Education, and Welfare publication No 74-1614. Government Printing Office, 1974

6. Ten-state Nutrition Survey 1968-1970, US Dept of Health, Education, and Welfare publication No (HSM) 72-8132. Government Printing Office, 1972

7. IJ Rimm and AA Rimm, Prev Med 3: 543-572, 1974

8. PB Goldblatt, ME More, and AJ Stunkard, J Am Med Assoc 192: 97-102, 1965

9. SM Garn and AS Ryan, Ecol Food Nutr 10: 237-239, 1981

10. SM Garn, SM Bailey, and ITT Higgins in Childhood Prevention of Atherosclerosis and Hypertension. RM Lauer and RB Shekelle, Editors, pp 187-204. Raven Press, New York, NY, 1980

11. SM Garn, SM Bailey, and PE Cole in Nutrition, Physiology, and Obesity. R Schemmel, Editor, pp 51-78. CRC Press, Palm Beach, FL, 1980

12. SM Garn, M LaVelle, and JJ Pilkington, Marr Fam Rev 7: 33-47, 1984

13. SM Garn, SM Bailey, and PE Cole, Am J Clin Nutr 32: 2375-2377, 1979 
14. P Biron, JG Mongeau, and D Bertrand, J Pediatr 91: 555-558, 1977

15. SM Garn, SM Bailey, and PE Cole, Am J Phys Anthropol 45: 539-543, 1976

16. AJ Stunkard, TIA Sorensen, C Hanis, TW Teasdale, R Chakraborty, WJ Schull, and F Schulsinger, N Engl J Med 314: 193-198, 1986

17. S Abraham, MD Carroll, MF Najjar, and R Fulwood, Vital and Health Statistics, Series II, No 230, US Dept of Public Health Service publication No 83-1680. Government Printing Office, 1983

18. SM Garn, WR Leonard, and KR Rosenberg, Am J Clin Nutr 44: 996-997, 1986

19. A Hartz, E Giefer, and AA Rimm, Ann Hum Genet 41: 185-193, 1977

20. C Bouchard, R Savard, JP Deprés, A Tremblay, and C Leblanc, Hum Biol 57: 61-75, 1985

21. AJ Stunkard, TT Foch, and Z Hrubek, J Am Med Assoc 256: 51-54, 1986

22. E Mason, Vet Rec 86: 612-616, 1970

23. SM Garn and JJ Pilkington, Ecol Food Nutr 15: 7-12, 1984
24. FE Johnston and RW Mack, Am J Dis Child 132: 862-864, 1978

25. PM Zack, WR Harlan, PE Leaverton, and J Cornoni-Huntley, J Pediatr 95: 126-130, 1979

26. SM Garn and M LaVelle, Am J Dis Child 139: $181-185,1985$

27. SM Garn, Curr Probl Pediatr 15 (2): 1-47, 1985

28. SM Garn, KR Rosenberg, and ITT Higgins, Ecol Food Nutr 8: 1-2, 1979

29. B Sherman, R Wallace, J Bean, and L Schlabaugh, J Clin Endocrinol Metab 52: 488-493, 1981

30. SM Garn, M LaVelle, KR Rosenberg, and V Hawthorne, Am J Clin Nutr 43: 879-883, 1986

31. WH Mueller, Yrbk Phys Anthrop 26: 215-230, 1983

32. JL Annest, CF Sing, P Biron, and JG Mongeau, Am J Epidemiol 117: 492-506, 1983

33. Obesity: A Report of the Royal College of Physicians, J Coll Physicians Lond 17: 3-65, 1983 